\title{
Modelos falsos en la ciencia: un valioso recurso para la comprensión de los fenómenos*
}

False Models in Science: A Valuable Resource for the Understanding of Phenomena

Antonio Diéguez ${ }^{\dagger}$

\begin{abstract}
Resumen
En las ciencias basadas en modelos, como la biología, los modelos desempeñan un papel explicativo fundamental e imprescindible. En los últimos años, algunos autores han señalado que la noción de 'comprensión' (understanding) ${ }^{1}$ puede arrojar alguna luz en el análisis de la explicación científica basada en modelos. Esta noción ha atraído una creciente atención en la filosofía de la ciencia y, en particular, en la filosofía de la biología. Tres preguntas centrales se han planteado en el debate que ha surgido al respecto: (1) ¿Qué es la comprensión científica?, (2) ¿es "factiva" (factive) la comprensión, es decir, presupone o implica la verdad de las creencias involucradas?, y (3) ipuede ser objetiva la comprensión? En este trabajo me centraré en la cuestión (2) y asumiré para ello la respuesta a la cuestión (1) ofrecida por Catherine Elgin. Defenderé que la comprensión no es factiva en lo que respecta al uso de modelos falsos -no implica aceptar como verdaderas las creencias involucradas. Distinguiré cuatro tipos de modelos falsos según el papel que la falsedad juega en su función explicativa y argumentaré que estos modelos falsos son herramientas muy útiles para la comprensión de los fenómenos y que su uso es frecuente en biología.
\end{abstract}

Palabras clave: comprensión científica - explicación - modelos científicos - modelos falsos

\begin{abstract}
In model-based sciences, like biology, models play a fundamental and indispensable explanatory role. In recent years, some authors have pointed out that the notion of understanding could shed light on the analysis of scientific explanation based on models. This notion has attracted growing attention in philosophy of science, particularly in philosophy of biology. Three central questions have been raised in the debate: (1) What is scientific understanding?; (2) is understanding factive, i.e., does understanding presuppose or imply truth of the beliefs involved?; and (3) can understanding be objective? In this paper I will focus on question (2), taking as an assumption the answer to question (1) offered by Catherine Elgin. I will argue that understanding is not factive when considering the use of false models it does not imply accepting the beliefs involved as true. I will distinguish four types of false models according to the role that falsity plays in their explanatory function and I will argue that these false models are very useful tools for the understanding of phenomena and that their use is frequent in biology.
\end{abstract}

Keywords: scientific understanding - explanation - scientific models - false models

\footnotetext{
* Recibido: 22 de Marzo de 2016. Aceptado con revisiones: 5 de Diciembre de 2016.

† Universidad de Málaga. Para contactar al autor, por favor, escribir a: dieguez@uma.es.

${ }^{1}$ Aunque puede haber otras alternativas, es habitual en la tradición filosófica traducir por 'comprensión' el término alemán 'Verstehen' y el término inglés 'understanding'. Sobre la dicotomía 'explicación/comprensión” en las ciencias, puede verse el clásico de von Wright (1980).

2 El término 'factive' es un neologismo en inglés, y no se identifica con 'factual', por eso hemos utilizado aquí un neologismo en español como es 'factivo'.

Metatheoria 8(1)(2017): 95-105. ISSN 1853-2322.

(C) Editorial de la Universidad Nacional de Tres de Febrero. Publicado en la República Argentina.
} 


\section{Introducción}

Algunos autores (p.e., Knuuttila \& Merz 2009, Leonelli 2009) han mostrado que la estrecha conexión existente entre explicación y comprensión es particularmente visible y reveladora en el uso explicativo de modelos. Dado el carácter lingüístico de la mayor parte de las explicaciones científicas, puede defenderse que la comprensión de los fenómenos es un objetivo más directo que la explicación en la construcción de modelos científicos. Desde este punto de vista, la comprensión de los fenómenos no sólo no es un subproducto de la explicación, sino que, al menos en ciertos casos, si podemos elaborar una explicación es sólo porque un modelo nos permite explorar y comprender los detalles de un fenómeno particular. La mera comprensión de un fenómeno a través de un modelo puede ser considerada en ocasiones como una explicación, como sucede con algunos modelos en genética de poblaciones. Podría decirse en tales casos que el modelo explica porque proporciona una comprensión genuina del fenómeno. De este modo, la comprensión sería el objetivo primigenio y la explicación sería un resultado del cumplimiento de dicho objetivo. Pero sea así o no en otros casos, es evidente que la mejora de nuestra comprensión es un fin básico explícitamente buscado cuando se usa un modelo con un propósito explicativo.

Dado que la noción de comprensión ha despertado un extenso debate y que han sido diversas las propuestas para su caracterización, conviene que aclaremos cómo vamos a entender aquí dicho concepto. No pretendo añadir una nueva definición a las ya existentes. Creo que la que ha propuesto Catherin Z. Elgin es, por razones diversas, bastante apropiada. Es elegante, abarcadora y clarificadora. Por lo tanto, es la que asumiré en este trabajo. Ella lo enuncia así:

La comprensión es la captación (grasp) de un cuerpo general amplio de información que está basada en los hechos, es sensible a la evidencia y nos capacita para realizar inferencias no triviales, argumentos, y quizás acciones relacionadas con el asunto al que la información pertenece. (Elgin 2009, p. 327) ${ }^{3}$

Una de las principales virtudes de esta caracterización es que resulta de utilidad para ver cómo los modelos científicos son usados con funciones explicativas y cómo este uso nos permite comprender los fenómenos, puesto que incluye tanto los aspectos teóricos como prácticos de la comprensión científica. Por un lado, la comprensión es descrita como una captación mental de información que nos permite inferir nuevas consecuencias acerca de los fenómenos. Por otro, este logro mental hace posible actuar sobre dichos fenómenos, incluyendo su manipulación. Ambos aspectos se consideran como fuertemente interconectados en la estrategia de las ciencias basadas en modelos (Godfrey-Smith 2006).

\section{Una tipología de modelos falsos}

Vayamos, pues, a la cuestión de si la comprensión es o no factiva. Uno de los autores que ha defendido una respuesta afirmativa es Jonathan Kvanvig (2003, cap. 8). Para él, decir que una persona comprende $p$ exige que $p$ sea verdadero. Él distingue entre comprensión proposicional y comprensión objetual. La primera consiste en comprender que algo es el caso o, en otras palabras, consiste en comprender una proposición que describe algo. La segunda consiste en comprender un objeto (un fenómeno, una situación, un lenguaje, etc.). Según Kvanvig, para comprender una proposición, ésta ha de ser verdadera, y de forma similar, para comprender un objeto, las creencias sobre el objeto han de ser en su mayor parte verdaderas.

No obstante, una afirmación así no casa bien con el uso frecuente que se hace de modelos falsos en la ciencia con vistas a la comprensión de los fenómenos. La expresión 'modelos falsos' sigue despertando aún recelos, y ello a pesar de su amplio uso en la literatura filosófica. Los modelos no

\footnotetext{
${ }^{3}$ El texto original dice asi: "Understanding is a grasp of a comprehensive general body of information that is grounded in fact, is duly responsive to evidence, and enables non-trivial inference, argument, and perhaps action regarding that subject the information pertains to".
} 
parecen ser ni verdaderos ni falsos en sentido estricto. En primer lugar, normalmente no son entidades lingüísticas, y en su uso formal y riguroso 'verdadero' y 'falso' son predicados metalingüísticos aplicables, por tanto, sólo a entidades lingüísticas. En segundo lugar, los modelos en sí mismos no parecen afirmar nada acerca de la realidad a menos que se les añada lo que Ronald Giere (1999) llamó 'hipótesis teóricas'. Estas hipótesis declaran que el sistema real es similar al modelo en algunos aspectos y en cierta medida. Son ellas, pues, las que serían verdaderas o falsas, y no los modelos como tales. Y, en tercer lugar, por lo general los modelos presentan numerosas idealizaciones (y abstracciones) que dejan de lado o distorsionan importantes aspectos del mundo real, lo cual hace de cualquier sistemamodelo algo inevitablemente ficticio. Así, el sistema hipotético descrito por un modelo científico suele ser irreal e idealizado. No hay gases ideales, péndulos perfectos, poblaciones completamente aisladas de predadores y presas, o poblaciones infinitas de individuos que se cruzan entre sí de forma completamente aleatoria. Tales cosas son sólo entidades hipotéticas imaginarias pero útiles, es decir, constructos ficticios creados para la investigación. Ciertamente, podemos generar con ellos algunas "verdades ficticias", y en este sentido algunas proposiciones son verdaderas o falsas dentro del modelo (p.e. en el modelo copernicano, el enunciado 'las órbitas planetarias son circulares' es verdadero). Pero estas verdades ficticias no son verdades literales sobre el mundo real (véase Godfrey-Smith 2009, Frigg 2010, Contessa 2010).

Sin embargo, los modelos pueden ser juzgados como verdaderos o falsos en un sentido más amplio e indirecto. De forma análoga a lo que sucede con los mapas, los modelos pueden ser interpretados como representaciones más o menos precisas o ajustadas de rasgos del mundo real. Un mapa es una representación parcial, simplificada, convencional, situada en una perspectiva, históricamente contingente e indefinidamente perfectible, de un territorio y de algunos de sus ítems geográficos (vías de ferrocarril, carreteras, restaurantes, monumentos, etc.). Un modelo es también una representación de alguna otra cosa; representa un sistema real. Una vez que tomamos en cuenta las convenciones de lectura vigentes y los diferentes fines e intereses que los cartógrafos pueden haber tenido en mente, un buen mapa debe mostrar algunas similitudes estructurales contextualmente útiles con el territorio representado, y debe preservar las relaciones significativas entre sus partes o elementos. Estas relaciones preservadas hacen posible que los razonamientos subrogatorios realizados a partir del mapa (tales como 'si estoy en Toledo, entonces debo tomar esta carretera para llegar a Madrid') conduzcan a las consecuencias correctas en relación con las características reales del territorio. Si esta condición no es satisfecha en un grado adecuado, no es extravagante afirmar que el mapa es desorientador, inexacto, incorrecto, o -al menos en los casos extremos- simplemente falso. Por el contrario, si se cumple la condición de la preservación de las relaciones estructurales, el mapa puede ser estimado como suficientemente exacto o aproximadamente verdadero (véase Kitcher 2001, cap. 5, y, para una opinión contraria, Sismondo \& Chrisman 2001). Del mismo modo, teniendo en cuenta el propósito con el que los científicos elaboran los modelos, es de esperar que un modelo proporcione descripciones fiables de algunas propiedades interesantes y relevantes en un determinado contexto del sistema real representado. Por lo tanto, un modelo muy poco realista, incompleto o contrafáctico, un modelo que no comparta suficientes propiedades relevantes con el sistema representado, o que fracase en su referencia a sus propiedades reales, podría ser considerado como falso (para una discusión y algunas precisiones importantes, véase Chakravartty 2010, Mäki 2011). De hecho, debido a las idealizaciones contenidas en muchos modelos, algunos autores piensan que dichos modelos no pueden ser si no falsos. Los modelos científicos altamente idealizados son falsos en el sentido de que no ofrecen -y no pueden ofrecer- una representación precisa del sistema real representado.

Esta idea necesita, sin embargo, de mayor cualificación, puesto que es bien conocido que a cada instante los científicos derivan de estos modelos altamente idealizados consecuencias útiles y suficientemente verdaderas acerca de las características y comportamiento de los sistemas representados. Lo interesante aquí es que los modelos falsos, bajo ciertas circunstancias, pueden ser usados para obtener una comprensión reveladora de los mecanismos de funcionamiento o de las causas de los fenómenos reales. Una adecuada comprensión de esos modelos falsos conduce a una adecuada comprensión del objeto o sistema representado. Algunos autores, como Hindriks (2008), 
Morrison (2009a) y Kennedy (2012) son más radicales y han argumentado -de forma convincente en mi opinión- que estos modelos explican en virtud de sus inexactitudes y falsedades, y no a pesar de ellas.

Creo que una forma útil de ver cómo estos modelos falsos pueden facilitar la comprensión de los fenómenos consiste en distinguir varias modalidades dentro de ellos, para analizar de forma separada sus peculiaridades como modelos explicativos. Porque, en efecto, los modelos pueden ser falsos en diversas formas y esto no es irrelevante en su uso. En lo que sigue, esbozaré una clasificación que no pretende ser exhaustiva, pero que creo que puede aportar bastante claridad a este complejo asunto. ${ }^{4}$ Distinguiré entre (1) modelos ajustables, (2) modelos plantilla, (3) modelos no-denotativos y (4) modelos contrastantes. Veamos sus características y sus diferencias.

(1) Modelos ajustables: Son modelos falsos susceptibles de mejoramiento mediante un proceso de des-idealización o concretización conducente a una descripción más precisa y realista de los fenómenos en circunstancias específicas. El modelo de Volterra para una población de depredadores y presas es un buen ejemplo. Dicho modelo afirma que la dinámica de interacciones entre dos especies en ese tipo de población puede ser adecuadamente descrita por dos simples ecuaciones. Si $D$ es la densidad de la población de depredadores y $P$ es la densidad de la población de presas, la tasa de cambio de las presas sería:

$$
d P / d t=r P-a P D
$$

y la tasa de cambio de los depredadores sería:

$$
d D / d t=h a P D-m D,
$$

donde $r$ es la tasa instantánea de crecimiento per capita en la población de presas, a es la medida de la tasa de capturas por cada depredador, $h$ es la medida de la eficiencia en la transformación de la energía obtenida por cada presa capturada en la producción de nuevos depredadores y $m$ es la tasa específica de mortalidad o migración de los depredadores. Este elegante modelo efectúa diversas idealizaciones. Supone que la única restricción al crecimiento de la población de presas es la existencia de depredadores, de modo que, en ausencia de los mismos, la población de presas crecería indefinidamente, lo cual es bastante irreal. Es fácil corregir esta suposición introduciendo un nuevo factor en las ecuaciones. Sea $K$ la capacidad de carga del medio, es decir, el tamaño máximo de la población de presas que ese medio puede sustentar, entonces la ecuación de la tasa de cambio de la población de presas sería:

$$
d P / d t=r P(1-P / K)-a P D
$$

Este nuevo factor introducido sustituye un crecimiento exponencial por un crecimiento logístico mucho más realista. Y esta sustitución conduce a cambios importantes en el comportamiento dinámico del sistema; a saber, las constantes oscilaciones del tamaño de las poblaciones de presa y depredador que se producen con el primer sistema de ecuaciones son amortiguadas con el tiempo hasta alcanzar un estado estacionario, o simplemente desaparecen. Y pueden realizarse correcciones similares para tomar en consideración el hecho simple de que cualquier depredador tiene un límite en su tasa de capturas (véase Rodríguez 1999, p. 274-287).

(2) Modelos plantilla: Son modelos que describen una situación ideal no existente en la realidad de la cual se desvían en cierto grado los sistemas reales debido a la influencia de diversos factores causales que deben ser determinados empíricamente en cada caso concreto, y es en la posibilidad que abre para realizar tal determinación en donde reside la utilidad del modelo. El modelo de Hardy-Weinberg encaja bien dentro de esta modalidad. Dicho modelo establece que la frecuencia genética de una

\footnotetext{
${ }^{4}$ Los tres primeros items de esta tipología pueden ser considerados en cierto modo como una simplificación de la clasificación que hace Wimsatt (1987) de los modelos falsos.
} 
población panmíctica e infinita no sujeta a selección natural, a mutación, o a migración, permanece constante a lo largo de las generaciones. Supongamos que hay dos alelos posibles para un cierto locus en el acervo genético de una población así; el alelo $A$, con una frecuencia inicial $p$, y el alelo $a$, con una frecuencia q. Siendo así, las frecuencias en el estado de equilibrio de los genotipos AA, Aa y aa serán $p^{2}$, $2 p q$ y $q^{2}$, respectivamente. Ninguna población real cumple los requisitos exigidos, pero precisamente lo que el modelo pretende poner de relieve es el hecho de que cualquier desviación que se encuentre con respecto a las frecuencias en el equilibrio en las frecuencias génicas realmente medidas debe ser explicada mediante la intervención de uno o más de los factores que han sido excluidos del modelo (acción de la selección natural, mutación, migración, deriva genética debido al pequeño tamaño de la población, emparejamientos no aleatorios, etc.). En este caso, carecería de sentido des-idealizar el modelo, puesto que su función no es entender el funcionamiento de un sistema real en una situación simplificada, sino fijar el punto más allá del cual debe tomarse en cuenta la acción de una fuerza evolutiva. Como Sober (1984, p. 23) explicó en su momento, este modelo describe un "estado de fuerza-cero". Otro ejemplo ilustrativo de este tipo de modelos es el modelo de proporción de sexos de R.A. Fisher. El modelo de Fisher explica por qué, bajo una gran variedad de circunstancias, la proporción estable (adaptativa) de machos y hembras en una población biológica es 1:1. Si la proporción real de sexos en una población se separa de esta proporción, debe entonces haber una causa para ello que ha de ser encontrada. Las poblaciones de ciertas especies de parásitos invertebrados, por ejemplo, contienen habitualmente un número de hembras mayor que el de machos. Estas poblaciones viven en hábitats aislados (el organismo huésped), en los que la mezcla con otras poblaciones es muy rara, y los individuos han de reproducirse muy rápidamente. La competición por el emparejamiento es, pues, meramente local, esto es, se da sólo contra otros individuos rivales que están en el organismo huésped. En tales circunstancias, la acción de la selección natural favorece una proporción de sexos sesgada hacia el lado femenino. Lo mismo sucede con una especie poliginia, el ciervo rojo Cervus elaphus. En esta especie, las hembras dominantes están mejor alimentadas y su descendencia es criada de forma que posee una fortaleza por encima de la media. Estas hembras dominantes tienden a tener más descendientes masculinos que femeninos (Ridley 1996, p. 307-312).

(3) Modelos no-denotativos: Son modelos irremediablemente falsos, puesto que postulan entidades, propiedades o mecanismos carentes por completo de referencia. No hay nada en el sistema real que se intenta representar con ellos que pueda ser designado como elemento denotado por los principales elementos del modelo. Por esta razón, a diferencia de los dos tipos previamente mencionados, estos modelos no pueden ser considerados ni siquiera como aproximadamente verdaderos. A veces, pero no siempre, tales modelos son usados únicamente para "salvar los fenómenos", como el modelo de epiciclos de Ptolomeo. Otras veces, como en el caso del calórico, del éter electromagnético, o del modelo hipocrático del equilibrio humoral como base de la salud, simplemente fracasan en su referencia a los rasgos reales de los fenómenos. Este tipo de modelos ha permanecido más desatendido que los anteriores en su análisis. Tres excepciones notables e interesantes son Morrison (2009b), Elgin (2010) y Toon (2010). Morrison estudia con detalle el modelo de éter de Maxwell como un ejemplo ilustrativo de cómo un mecanismo ficticio puede proporcionar información y dar lugar a predicciones útiles. Afirma que hay varias formas en las que estos modelos pueden desempeñar su tarea y, por tanto, se necesita un análisis cuidadoso en cada caso. Elgin cree que estos modelos no deben ser considerados como ficticios (fictive), en el sentido en que lo es el modelo de gas ideal, sino como defectuosos (defective), porque, a diferencia de los modelos que usan ficciones para intentar denotar algún objeto real, estos modelos no referenciales se proponen denotar algo en un sistema real, pero de hecho tal sistema no existe en el mundo. En el caso de los modelos que usan ficciones, hay algún sistema real que comparte con el modelo algunas de las propiedades ejemplificadas por él. Pero esto no sucede con los modelos no-denotativos. Éstos no pueden compartir ninguna propiedad con el sistema que se intenta representar por la sencilla razón de que dicho sistema no existe en absoluto. Por su parte, Toon sostiene que no siempre estos modelos intentan representar algún sistema real; a veces el sistema que se intenta representar es explícitamente un objeto no existente, como, por ejemplo, en el caso del modelo 
de un puente aún no construido, o de un modelo de bolas y alambre de alguna configuración atómica irreal.

(4) Modelos contrastantes: Con algunas excepciones en el último caso mencionado, los tres tipos de modelos falsos que hemos distinguido hasta ahora son construidos con la intención de representar (algún aspecto de) un sistema real, aunque esta intención fracase en el caso de los modelos nodenotativos. Pero hay otro tipo de modelos falsos que no se elaboran con esa intención. Se trata de modelos explícitamente formulados para representar un sistema puramente imaginario, sin contrapartida real conocida. No habría ningún sistema real que pudiera ser considerado como representado, si quiera aproximadamente, por ellos. Pero, aunque los sistemas representados por este tipo de modelos sean completamente ficticios, son propuestos, sin embargo, para entender algunos fenómenos reales. En ocasiones, estos modelos pueden incluso estar en conflicto con leyes científicas; y, a diferencia de lo que sucede con otros modelos basados en sistemas no existentes (gas ideal, péndulo perfecto, población infinita, etc.), en este caso es la disimilitud o el contraste entre el sistema modelo y los fenómenos reales los que llevan la carga de la función explicativa y arrojan luz sobre el comportamiento de dichos fenómenos. Como escribe Michael Weisberg, "en la medida en que podemos entender porqué no existen [los fenómenos descritos por el modelo], podemos haber obtenido una mejor comprensión de los fenómenos que sí existen” (Weisberg 2007, p. 223). Por esa razón, estos modelos pueden designarse como 'modelos contrastantes' (Diéguez 2013). Un buen ejemplo sería, en mi opinión, el modelo de Laurence D. Hurst (1996) para explicar porqué normalmente sólo hay dos sexos en las especies biológicas. Hurst ofrece un modelo matemático para una población de protistas isógamos en la que hay tres tipos sexuales (mating type). El modelo muestra, entre otras cosas, que a menos que el coste de encontrar pareja sea alto, esta población y otras poblaciones de organismos con fusión de gametos debe evolucionar hace dos tipos sexuales, y por tanto, que los organismos con fusión de gametos y más de dos sexos han ser raros en la naturaleza. Más recientemente, y en la misma línea, Tamás Czárán y Rolf Hoekstra (2004) han construido un modelo que muestra que, en las condiciones habituales, "una población que tenga dos tipos sexuales puede desplazar a una población pansexual que sea similar en todos los demás aspectos” (p. 7). Una especie o población pansexual sería aquella en la que cualquier célula sexual de los organismos pudiera fusionarse con cualquier otra célula sexual. De forma análoga, un modelo desarrollado por Michael Bonsall (2006) indica que, a menos que se introduzcan diversas y complejas innovaciones fisiológicas, los cigotos diploides poseen una eficacia biológica mayor que los cigotos triploides (i.e., los cigotos producidos por la fusión de tres tipos de gametos). En estos tres ejemplos, los modelos propuestos usan tres o más tipos sexuales. Con la excepción de los hongos y algunos raros organismos, ${ }^{5}$ esto es una situación bastante extraña en la naturaleza, de modo que estos modelos no buscan encajar con ningún sistema real. Sin embargo, muestran que los sistemas irreales modelados son inestables o tienen una eficacia biológica menor que los sistemas con dos sexos. En tal sentido, estos modelos proporcionan alguna comprensión en lo que concierne a la extendida existencia de este último tipo de modalidad reproductiva. Desde el punto de vista del uso explicativo, estos modelos divergen de otros en un aspecto importante: lo que podríamos designar como su 'intención representacional' no es la misma. Un gas ideal es una idealización de ciertas entidades reales: los gases normales. Como modelo, el gas ideal intenta representar a cualquier gas real en un contexto real. Por el contrario, una especie trisexual no es una idealización de ninguna especie real, sino una ficción no accesible por medio de simplificaciones o abstracciones realizadas sobre sistemas reales.

\section{3. ¿Cómo tiene lugar la comprensión mediante modelos falsos?}

Ahora estamos en disposición de retornar a la cuestión de la "factividad" de la comprensión científica. En primer lugar, debemos distinguir entre la comprensión de un fenómeno por medio de un modelo y

\footnotetext{
${ }^{5}$ El protozoo ciliado Tetrahymena thermophile es uno de estos raros organismos. Posee siete tipos sexuales. Algunos insectos eusociales, como las hormigas del género Pogonomyrmex, poseen un sistema reproductivo con tres sexos.
} 
la comprensión del modelo mismo. Cuando consideramos el caso de la comprensión de un modelo como tal, la comprensión parece claramente no-factiva. Obviamente, podemos entender modelos falsos, p.ej. el modelo ptolemaico de los movimientos planetarios puede ser comprendido por cualquier estudiante aplicado de la historia de la astronomía. De forma análoga, podemos entender también proposiciones falsas, como 'España es una isla', o proposiciones que no son literalmente verdaderas, sino sólo "verdades en la ficción”, como "los libros de caballería volvieron loco a Don Quijote', o 'todos los vórtices en el éter electromagnético giran en la misma dirección’. Además de esto, podemos entender qué es un péndulo ideal o qué es un gas ideal, aunque no haya péndulos ideales ni gases ideales. Esto es un hecho trivial admitido por los que defienden la factividad (o quasi-factividad) de la comprensión. Lo que éstos suelen subrayar es que las cosas no son así cuando volvemos la mirada a la comprensión de una situación objetiva y un fenómeno. En este caso, parece que el fenómeno a comprender no puede ser un fenómeno espurio. Siguiendo la sugerencia de Kvanvig (2003), no sería posible comprender que $p$ es el caso si $p$ no es de hecho el caso. Sería absurdo decir que 'Juan comprende por qué el azúcar nunca se disuelve en agua' o que 'María comprende cómo la danza de la lluvia produce lluvia'. Parece claro también que no se puede entender la causa de algo si las creencias sobre esa causa son completamente falsas. No puedo comprender por qué se ha estropeado el coche que conduzco sí creo erróneamente que la causa es un defecto en el carburador cuando la causa real es un problema con los cables de las bujías. Y, lo que importa más aquí, como han mantenido algunos autores, la comprensión de los modelos falsos no implica la comprensión de los fenómenos modelados. "Se puede comprender -escribe Kvanvig (2009, p. 342)- el modelo o la teoría misma, como cuando comprendemos la teoría del flogisto. Sin embargo, no por ello comprendemos así la combustión”.

Ahora bien, demandar factividad a cualquier forma de comprensión sería una exigencia demasiado fuerte. A pesar de la afirmación de Kvanvig, a veces comprendemos por medio de modelos que contienen un gran número de supuestos falsos el comportamiento de sistemas reales -como un gas- o de sistemas imaginarios -como una especie animal con tres sexos- ${ }^{6} \mathrm{Y}$ en este uso de los modelos, las falsedades no son ni periféricas ni dispensables. Veamos ahora con más detalle cómo pueden realizar esta función los diferentes tipos de modelos falsos que he distinguido anteriormente.

Los modelos ajustables son representaciones idealizadas y abstractas de los sistemas que son su objeto. No obstante, estas idealizaciones y abstracciones, como se ha explicado, no son impedimentos para la correcta comprensión de los fenómenos, sino más bien herramientas para conseguirla. Los modelos ajustables nos permiten, por ejemplo, prever cómo el sistema objeto cambiaría si las condiciones iniciales fueran diferentes. Nos proporcionan algunas respuestas relevantes a las demandas de información contrafáctica acerca del comportamiento del sistema modelado en una variedad de circunstancias. Por otro lado, como ha señalado Elgin (2004), estos modelos ejemplifican algunas de las propiedades más significativas del sistema representado, y nos facilitan el análisis de las relaciones e interacciones entre dichas propiedades. Al respecto, Elgin escribe:

\begin{abstract}
Ningún gas real tiene las propiedades de un gas ideal. El modelo es, sin embargo, clarificador, porque comprendemos las propiedades de los gases reales en términos de su desviación del ideal. En tal caso, la comprensión implica un patrón de esquemas y correcciones. Representamos los fenómenos con un modelo esquemático e introducimos las correcciones necesarias para hacerlo concordar con los hechos. Se necesitan diferentes correcciones para hacer concordar el comportamiento de diferentes gases. El ideal ficticio, por tanto, funciona a modo de mínimo común denominador para facilitar el razonamiento sobre, y la comparación de, gases reales. Primero 'despejamos' el caso simple, después introducimos las complicaciones que sean necesarias. (Elgin 2004, pp. 126-127)
\end{abstract}

Así pues, los modelos ajustables conducen a una mejor comprensión de los fenómenos reales en la medida en que los procesos de des-idealización y concretización dan lugar a mejores predicciones y análisis. Estos procesos, por el contrario, no son relevantes en el caso de los modelos plantilla, a pesar

\footnotetext{
${ }^{6}$ Quizás sea oportuno hacer notar que esto no contradice la asunción que hemos hecho aquí de la noción de comprensión defendida por Elgin. Estos modelos, aunque sean falsos, o contengan falsedades, o fracasen en la referencia, siguen siendo modelos que captan información basada en hechos, son sensibles a la evidencia empírica y permiten realizar inferencias acerca de la realidad.
} 
de que éstos proporcionan comprensión de una forma parecida a los anteriores. Los modelos plantilla son usados como un recurso para detectar las razones de la separación de la situación ideal por parte de los sistemas reales. En la medida en que estas razones son descubiertas, alcanzamos una mejor comprensión de las circunstancias que están implicadas en el funcionamiento del sistema real representado, así como de los factores que lo modifican y lo configuran. En cuanto a los modelos contrastantes (dejo los no-denotativos para el final), funcionan de forma diferente, pero no muy diferente. A diferencia de los modelos ajustables -y en cierto sentido también de los modelos plantilla-, en los que es la similitud con el sistema real lo que resulta interesante, en el caso de los modelos contrastantes es la disimilitud, la disparidad, lo que porta la carga explicativa y arroja luz sobre el funcionamiento del sistema real. Estos modelos responden a la pregunta contrastante 'ipor qué $p$ en lugar de q?', y así, por ejemplo, nos permiten entender por qué hay dos sexos en lugar de haber más. ${ }^{7}$

Los modelos no-denotativos presentan mayores dificultades. Es una cuestión controvertida si estos modelos son capaces o no de proporcionar algún tipo de comprensión. Por un lado, puede argüirse que no constituyen una base suficiente para una genuina comprensión. Se trataría simplemente de casos de mala comprensión, de interpretaciones equivocadas (misunderstanding). Es cierto que la astronomía de Ptolomeo fue útil para la navegación y para la predicción de eclipses, pero de hecho los epiciclos no están ni siquiera remotamente conectados con los mecanismos que causan realmente los movimientos planetarios. Por lo tanto, dichos epiciclos no pueden ofrecernos una comprensión genuina de estos movimientos. Y lo mismo puede aplicarse a la química del flogisto y al modelo del éter de Maxwell. Wimsatt (1987, p. 30) parece adoptar esta posición cuando escribe: “PProporcionará cualquier modelo falso un camino hacia la verdad? La respuesta es obviamente un enfático «ino!». Algunos modelos están tan equivocados, o sus incorrecciones son tan difíciles de analizar, que mejor buscamos en otra parte". Pero, por otro lado, puede también argüirse que este tipo de modelos es capaz de proporcionarnos una comprensión defectuosa o imperfecta, pero también en algún grado epistémicamente valiosa, de los fenómenos. En cualquier caso, siempre será preferible a la mera ignorancia. Desde un punto de vista instrumentalista o constructivista, estos modelos han hecho posible un cierto control sobre los fenómenos o han contribuido a estructurar algunos mundos posibles no carentes de interés, de modo que, aunque no puedan ser considerados como representaciones aproximadamente verdaderas de la realidad, han sido sin duda herramientas útiles en nuestro manejo cognitivo y práctico del mundo. Aparentemente, el conocimiento y la comprensión no siguen las mismas reglas epistémicas. Un "conocimiento falso" no es conocimiento en absoluto, puesto que el conocimiento ha de ser verdadero por definición. La comprensión, por el contrario, no parece ser un asunto tan dicotómico. Una comprensión falsa (una mala comprensión) puede ser de algún modo un cierto tipo comprensión incipiente o imperfecta, y podría ser visto en un momento ulterior como el primer paso incorrecto en el camino que condujo a la comprensión adecuada de los fenómenos.

Por lo tanto, un juicio equilibrado sobre el papel de los modelos no-denotativos exigiría en cada caso un riguroso y extenso análisis histórico. Por supuesto que no podemos hacer ese análisis aquí para ninguno de ellos, pero podemos recordar algunos hechos bien conocidos que ayudarán a contextualizar el asunto. Los modelos planetarios de Ptolomeo significaron un progreso real en la comprensión de la estructura del universo con respecto a los modelos de Eudoxo y Calipo o el modelo aristotélico de las esferas. Asumiendo que una de las principales características funcionales de un modelo científico es la de facilitar razonamientos subrogarorios acerca del sistema modelado (véase

\footnotetext{
Paul Humphrey ve aquí el límite para un uso explicativo de este tipo de modelos. Según su opinión, estos modelos podrían proporcionar comprensión, pero no explicación. Así, escribe: "aquí, por tanto, es quizás donde se sitúa una parte de la frontera entre explicación y comprensión. Aunque la exploración de modelos que violan las leyes de nuestro universo puede mejorar nuestra comprensión científica, tales modelos no pueden ser usados en explicaciones. Un ejemplo bien conocido implica las condiciones bajo las cuales la vida pudo emerger en el universo. Las preguntas 'ccómo pudo haber sido?' (how possibly?) que surgieron en la vecindad de los principios antrópicos añadieron cosas a nuestra comprensión de cómo la vida pudo haber emergido si las leyes hubieran sido distintas, pero las respuestas dadas no pueden explicar cómo surgió la vida en nuestro universo" (Humphrey 2006, pp. 42-43). No obstante, creo que los tres ejemplos que he mencionado antes muestran que los escrúpulos de Humphrey son exagerados y que estos modelos pueden desempeñar en ocasiones funciones explicativas.
} 
Swoyer 1991, Suárez 2004, Contessa 2007), hemos de admitir que los modelos de Ptolomeo cumplieron razonablemente esta función cuando fueron elaborados. Es posible -aunque algunos historiadores disienten al respecto- que fueran propuestos como meros modelos matemáticos para calcular las posiciones de los planetas, no como modelos físicos que intentaran representar los mecanismos reales del cosmos. Al menos así fueron comúnmente interpretados durante la Edad Media. Pero los modelos de Ptolomeo fueron capaces de generar algunas consecuencias acerca de los cambios en el brillo de los planetas, acerca de sus aparentes movimientos de retrogradación, acerca de sus variaciones en velocidad sobre el fondo de la eclíptica, acerca de la ausencia de paralaje estelar, etc.

Sin embargo, desde la perspectiva contemporánea, vemos a este conjunto de modelos como representaciones completamente falsas de la realidad, no como verdades aproximadas (para una opinión contraria, véase Niiniluoto 1999, p. 192), y tendemos a consideraros incapaces de proporcionar una comprensión genuina de los movimientos planetarios. No pueden responder a la mayor parte de las cuestiones que podrían suscitarse desde la perspectiva del conocimiento que tenemos actualmente acerca de dichos movimientos. La representación que hacen de esos movimientos difiere por completo de la nuestra. No podemos aceptar tal representación sin rechazar al mismo tiempo casi todo nuestro conocimiento científico acerca del Sistema Solar y de la física en general. Por ello, pocas personas estarían dispuestas a aceptar que los modelos de Ptolomeo proporcionan algún tipo de comprensión sobre el funcionamiento del universo.

Por su parte, al igual que los epiciclos, los humores hipocráticos, el flogisto o el calórico, el éter de Maxwell es una entidad ficticia (si bien algunos científicos, como el físico británico Oliver Lodge, creyó hasta cierto punto en su existencia real, como sucedió también con los epiciclos, el flogisto y el calórico). Pero probablemente somos menos reticentes que en los otros casos a admitir que jugó un papel fundamental en la comprensión de los fenómenos físicos. El modelo mecánico del éter electromagnético cumplió una función muy valiosa en la articulación de la teoría electromagnética de Maxwell. Por eso, desde nuestra actual perspectiva, es razonable pensar que su contribución al desarrollo y el progreso de dicha teoría justificaba su uso, independientemente del hecho de su fracaso a la hora de denotar un sistema real. Facilitó los cálculos, guio ulteriores investigaciones, tuvo un valor heurístico y fue una herramienta útil para el razonamiento subrogatorio y para derivar algunas relaciones matemáticas. El modelo del éter condujo a Maxwell, por ejemplo, a la conclusión de que la luz debía ser un cierto tipo de onda electromagnética, puesto que las ondas transversales serían transmitidas en el éter a la velocidad de la luz (véase Harman 1982, cap. 4; y véase también Chalmers 1986 para un punto de vista contrario). Pero Maxwell le atribuyó básicamente una función ilustrativa y auxiliar, sin que pretendiera que reflejara nada real, y finalmente su teoría -las ecuaciones del campo electromagnético- prescindía del modelo. ${ }^{8}$

¿Qué hace que sea tan diferente el modo en que vemos a los epiciclos de Ptolomeo del modo en que vemos al éter de Maxwell en relación con su papel en la comprensión de los fenómenos naturales? Creo que una buena sugerencia para contestar a esta pregunta se encuentra en la siguiente reflexión de Margaret Morrison:

Dado que muchos modelos no pueden ser evaluados sobre la base de su capacidad para proporcionar una representación realista, necesitamos centrar menos nuestra atención en la distinción entre modelos "heurísticos" y modelos "realistas", y en lugar de eso, hemos de subrayar el modo en que los modelos funcionan en el desarrollo de leyes y teorías. (Morrison 2005, p. 170)

Una diferencia clara entre los epiciclos de Ptolomeo y el modelo de Maxwell es precisamente que este último fue útil en el desarrollo de leyes y teorías que aceptamos en la actualidad, cosa que no sucede con el primero. De acuerdo con esto, puede afirmarse que comprendemos los fenómenos reales por medio de modelos no-denotativos sólo si estos modelos han sido útiles en el desarrollo de leyes o hipótesis que puedan ser justificadas por medio de teorías aceptadas en el momento presente.

\footnotetext{
${ }^{8}$ Aunque es posible que en ocasiones Maxwell jugara una doble estrategia. Debo este comentario a un evaluador anónimo.
} 


\section{Conclusiones}

Los modelos falsos son recursos muy eficaces para obtener una comprensión científica de los fenómenos naturales, y han cumplido un importante papel histórico en el desarrollo de la ciencia, particularmente en la biología, que suele ser considerada como una ciencia basada en modelos. Teniendo en cuenta las diferentes formas en que podemos perseguir esta meta, pueden distinguirse entre modelos ajustables, modelos plantilla, modelos no-denotativos y modelos contrastantes. El caso más complejo es el de los modelos no-denotativos, cuya capacidad para aportar una comprensión de los fenómenos no puede ser tratada mediante un análisis genérico, sino que debe atenderse a cada caso particular para poder juzgar. Todos ellos implican falsedades que son necesarias para la explicación del funcionamiento de los sistemas representados y para la comprensión de los fenómenos reales. Por lo tanto, la comprensión, a diferencia del conocimiento, no es factiva. No presupone que la mayoría de las creencias implicadas en el proceso de comprensión deban ser verdaderas.

\section{Bibliografía}

Bonsall, M.B. (2006), "The Evolution of Anisogamy: The Adaptive Significance of Damage, Repair and Mortality", Journal of Theoretical Biology 238: 198-210.

Chakravartty, A. (2010), "Truth and Representation in Science: Two Inspiration from Art", en Frigg, R. y M.C. Hunter (eds.), Beyond Mimesis and Conventions. Representation in Art and Science, Dordrecht: Springer, pp. 33-50.

Chalmers, A. (1986), “The Heuristic Role of Maxwell's Mechanical Model of Electromagnetic Phenomena”, Studies in History and Philosophy of Science 17(4): 415-427.

Contessa, G. (2007), "Scientific Representation, Interpretation, and Surrogative Reasoning", Philosophy of Science 74: 48-68.

Contessa, G. (2010), "Scientific Models and Fictional Objects", Synthese 172: 215-229.

Czárán, T.L. y R.F. Hoekstra (2004), “Evolution of Sexual Asymmetry”, BMC Evolutionary Biology 4(34): 1-12.

de Regt, H.W., Leonelli, S. y K. Eigner (eds.) (2009), Scientific Understanding. Philosophical Perspectives, Pittsburgh: University of Pittsburgh Press.

Diéguez, A. (2013), "When do Models Provide Genuine Understanding, and why Does It Matter?", History and Philosophy of the Life Sciences 35(4): 599-620.

Elgin, C.Z. (2004), "True Enough”, Philosophical Issues 14(1): 113-131.

Elgin, C.Z. (2009), "Is Understanding Factive?", en Haddock, A., Millar, A. y D. Pritchard (eds.), Epistemic Value, Oxford: Oxford University Press, pp. 322-329.

Elgin, C.Z. (2010), "Telling Instances", en Frigg, R. y M.C. Hunter (eds.), Beyond Mimesis and Conventions. Representation in Art and Science, Dordrecht: Springer, pp. 1-17.

Frigg, R. (2010), "Models and Fictions", Synthese 172: 251-268.

Giere, R. (1999), Science Without Laws, Chicago: University of Chicago Press.

Godfrey-Smith, P. (2006), “The Strategy of Model-based Science”, Biology $\mathcal{E}$ Philosophy 21: 725-740.

Godfrey-Smith, P. (2009), "Models and Fictions in Science", Philosophical Studies 143: 101-116.

Harman, F.M. (1982), Energy, Force, and Matter. The Conceptual Development of Nineteenth-Century Physics, Cambridge: Cambridge University Press.

Hindriks, F. (2008), "False Models as Explanatory Engines", Philosophy of the Social Sciences 38(3): 334-360.

Humphrey, P. (2006), "Invariance, Explanation, and Understanding", Metascience 15: 39-44.

Kennedy, A.G. (2012), "A Non-Representationalist View of Model Explanation", Studies in History and Philosophy of Science 43(2): 326-332. 
Kitcher, P. (2001), Science, Truth, and Democracy, Oxford: Oxford University Press.

Knuuttila, T. y M. Merz (2009), "Understanding by Modeling. An Objectivist Approach”, en de Regt, H.W., Leonelli, S. y K. Eigner (eds.), Scientific Understanding. Philosophical Perspectives, Pittsburgh: University of Pittsburgh Press, pp. 146-165.

Kvanvig, J. (2003), The Value of Knowledge and the Pursuit of Understanding, Cambridge: Cambridge University Press.

Kvanvig, J. (2009), "Responses to Critics”, en Haddock, A., Millar, A. y D. Pritchard (eds.), Epistemic Value, Oxford: Oxford University Press, pp. 339-353.

Leonelli, S. (2009), "Understanding in Biology: The Impure Nature of Biological Knowledge", en de Regt, H.W., Leonelli, S. y K. Eigner (eds.), Scientific Understanding. Philosophical Perspectives, Pittsburgh: University of Pittsburgh Press., pp. 189-209.

Mäki, U. (2011), "Models and the Locus of their Truth", Synthese 180: 47-63.

Morrison, M. (2005), “Approximating the Real: The Role of Idealizations in Physical Theory”, en Jones, M.R. y N. Cartwright (eds.), Idealization XII: Correcting the Model. Idealization and Abstraction in the Sciences (Poznań Studies in the Philosophy of the Sciences and the Humanities, vol. 86), Amsterdam-New York: Rodopi, pp. 145-172.

Morrison, M. (2009a), "Understanding in Physics and Biology", en de Regt, H.W., Leonelli, S. y K. Eigner (eds.), Scientific Understanding. Philosophical Perspectives, Pittsburgh: University of Pittsburgh Press, pp. 123-145.

Morrison, M. (2009b), "Fictions, Representations, and Reality", en Suárez, M. (ed.), Fictions in Science, New York: Routledge, pp. 110-135.

Niiniluoto, I. (1999), Critical Scientific Realism, Oxford: Oxford University Press.

Rodríguez, J. (1999), Ecología, Madrid: Pirámide.

Ridley, M. (1996), Evolution, $2^{\text {nd }}$ edition, Cambridge: Blackwell.

Sismondo, S. y N. Chrisman (2001), "Deflationary Metaphysics and the Natures of Maps", Philosophy of Science 68: S38. S49.

Sober, E. (1984), The Nature of Selection, Chicago: The University of Chicago Press.

Suárez, M. (2004), “An Inferential Conception of Scientific Representation”, Philosophy of Science 71: 767-779.

Swoyer, C. (1991), "Structural Representation and Surrogative Reasoning", Synthese 87: 449-508.

Toon, A. (2010), "Models as Make-Believe", en Frigg, R. y M.C. Hunter (eds.), Beyond Mimesis and Conventions. Representation in Art and Science, Dordrecht: Springer, pp. 71-96.

Von Wright, G.H. (1980), Explicación y comprensión, Madrid: Alianza.

Weisberg, M. (2007), "Who is a Modeler?", British Journal for the Philosophy of Science 58: 207-233.

Wimsatt, W.C. (1987), "False Models as Means to Truer Theories", en Nitecki, M.H. y A. Hoffman (eds.), Neutral Models in Biology, New York: Oxford University Press, pp. 23-55. 